\title{
Plasmonics: metallic nanostructures for energy guiding and sensing
}

\section{Stefan A. Maier}

Stefan A. Maier, "Plasmonics: metallic nanostructures for energy guiding and sensing," Proc. SPIE 5593, Nanosensing: Materials and Devices, (29 December 2004); doi: 10.1117/12.570803

SPIE. Event: Optics East, 2004, Philadelphia, Pennsylvania, United States 
Invited Paper

\title{
Plasmonics: Metallic Nanostructures for Energy Guiding and Sensing
}

\author{
Stefan A. Maier* \\ Thomas Watson Laboratory of Applied Physics, California Institute of Technology, Pasadena, CA
}

\begin{abstract}
We investigate the optical properties of arrays of closely spaced metal nanoparticles in view of their potential to guide electromagnetic energy with a lateral mode confinement below the diffraction limit of light. Finite-difference timedomain simulations of short arrays of noble metal nanospheres show that electromagnetic pulses at optical frequencies can propagate along the arrays due to near-field interactions between plasmon-polariton modes of adjacent nanoparticles. Near-field microscopy enables the study of energy transport in these plasmon waveguides and shows experimental evidence for energy propagation over a distance of $0.5 \mu \mathrm{m}$ for plasmon waveguides consisting of spheroidal silver particles fabricated using electron beam lithography.
\end{abstract}

Keywords: plasmon waveguides, metal nanoparticles, near-field optics, NSOM, surface plasmon, plasmon-polariton

\section{INTRODUCTION}

The miniaturization of optical devices to size dimensions akin to their electronic counterparts is a major goal of current research efforts in optoelectronics, photonics and semiconductor manufacturing. A high integration of optical components allowing the fabrication of all-optical chips for computing and sensing requires both a confinement of the guided optical modes to small dimensions and the ability to route energy around sharp corners. Current technologies that are driving a revolution in the fabrication of integrated optical components are planar waveguides, optical fibers and photonic crystals, which can confine and guide electromagnetic energy in spatial dimensions in the micron and submicron regime.

Whereas waveguides based on the principle of total internal reflection such as optical fibers do not allow for the guiding of light around sharp corners with a bending radius considerably smaller than the wavelength of light $\lambda^{1}$, engineering of defect modes in photonic crystals has enabled the fabrication of defect waveguides with complex guiding geometries ${ }^{2}$. The further integration of active devices such as defect mode lasers into photonic crystals ${ }^{3}$ will ensure a prominent spot for this technology in the creation of optical chips.

The size and density of optical devices employing these technologies is nonetheless restricted by the diffraction limit $\lambda / 2 n$ of light, which imposes a lower size limit of a few hundred nanometers on the optical mode size. Thus, a size mismatch between highly integrated electronic devices with lateral dimensions of a few tens of nanometers and optical guiding components persists and needs to be overcome.

The diffraction limit for the guiding of electromagnetic energy can be overcome if the optical mode is converted into a non-radiating mode, which can be confined to lateral dimensions smaller than the diffraction limit due to large inplane wave vectors upon resonant exciation. Prominent examples that have been the focus of intense research over the last couple of decades are surface plasmon-polaritons in metals. Plasmons are coherent oscillations of the conduction electrons of the metal against the static positive background of the metal ion cores. Whereas plasmons in bulk metal do not couple to light fields, a two-dimensional metal surface can sustain plasmons if excited by light either via evanescent prism coupling or the help of surface corrugations to ensure momentum matching ${ }^{4}$. Such surface plasmons propagate as coherent electron oscillations parallel to the metal surface and decay evanescently perpendicular to it. Thus, the electromagnetic energy is confined to dimensions below the diffraction limit perpendicular to the metal surface. Corrugations can further act as light-scattering centers for surface plasmons, allowing for the fabrication of interesting optical devices such as an all-optical transistor ${ }^{5}$. 
A further confinement of energy-guiding surface plasmon modes can be achieved using metal nanowires instead of extended surfaces. In nanowires, the confinement of the electrons in two dimensions leads to well-defined dipole surface plasmon resonances if the lateral dimensions of the wire are much smaller than the wavelength of the exiting light. Thus, the optical properties of metal nanowires can be optimized for particular wavelengths of interest, and non-regular cross sections and coupling between closely spaced nanowires allow a further tuning of the optical response ${ }^{6}$. Indeed, the propagation of electromagnetic energy has been demonstrated along noble metal stripes with widths of a few microns ${ }^{7}$ and along nanowires with subwavelength cross sections ${ }^{8}$, and propagation lengths of a few microns have been found. Related to this principle is the interesting idea of one-dimensional negative dielectric core waveguides, where metals instead of dielectric materials are used as the core in a waveguide with a cross section below the diffraction limit ${ }^{9}$.

Another intriguing nanoscale system that can sustain surface plasmons are metal nanoparticles, and their interaction with light has been the focus of intense research in recent years ${ }^{10,11}$. In metal nanoparticles, the three-dimensional confinement of the electrons leads to well-defined surface plasmon resonances at specific frequencies. From work on single noble metal nanoparticles, it is well established that light at the surface plasmon resonance frequencies interacts strongly with metal particles and excites a collective motion of the conduction electrons, or plasmon ${ }^{12}$. These resonance frequencies are typically in the visible or infrared part of the spectrum for gold and silver nanoparticles embedded in a variety of hosts. For particles with a diameter much smaller than the wavelength $\lambda$ of the exciting light, plasmon excitations produce an oscillating electric dipole field resulting in a resonantly enhanced non-propagating electromagnetic near-field close to the particle surface.

Recently, it has been suggested that near-field interactions between closely spaced metal nanoparticles in regular one-dimensional particle arrays can lead to the coherent propagation of electromagnetic energy along the arrays with lateral mode sizes below the diffraction limit ${ }^{13}$. For $\mathrm{Au}$ and $\mathrm{Ag}$ nanoparticles in air, group velocities for energy transport higher than the saturated electron velocities in semiconductors and energy decay lengths of a couple of hundred nanometers have been predicted. Furthermore, it was suggested that these so-called plasmon waveguides can guide electromagnetic energy around sharp corners and tee structures, and an all-optical modulator based on interference operating below the diffraction limit was proposed ${ }^{14}$.

The use of ordered arrays of metal nanoparticles as plasmon waveguides is intriguing from both a technology oriented and a fundamental point of view. Metal nanoparticles can be fabricated using a wide arsenal of tools including electron beam lithography ${ }^{15}$, colloidal synthesis ${ }^{16}$, self-assembly ${ }^{17}$, and ion irradiation ${ }^{18}$. Recently, we observed a preferential alignment of silver nanoclusters nucleated by $1 \mathrm{MeV} \mathrm{Xe}^{+}$radiation in a silver ion rich glass upon angular irradiation with a $30 \mathrm{MeV}$ silicon ion beam at $77 \mathrm{~K}$ (Figure $1,{ }^{19}$ ). Thus, ion beams show the potential for large volume fabrication of anisotropically distributed metal nanoparticles, and it is intriguing to investigate the optical properties of such arrays. Split plasmon bands showing red-shifts of the plasmon resonance due to particle interactions into the nearinfrared were observed ${ }^{19}$.

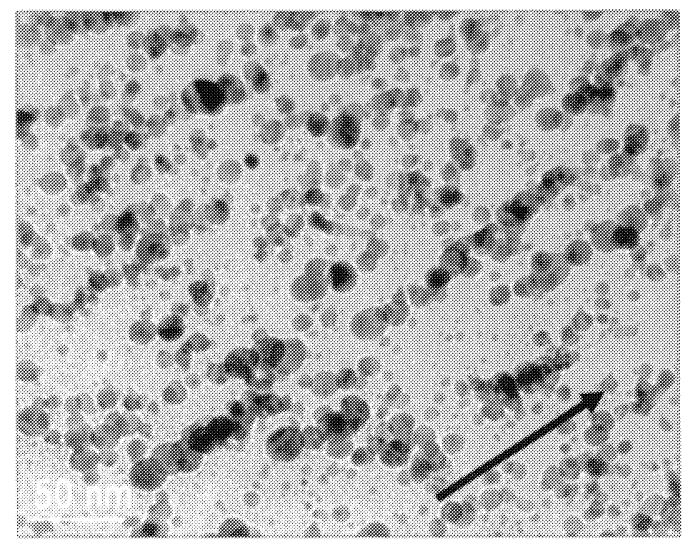

Figure 1. Plan view TEM image of a metal nanocluster sample under normal incidence. The sample was irradiated with $1 \mathrm{MeV}$ Xe and $30 \mathrm{MeV}$ Si. An alignment of the Ag nanoclusters in the beam direction indicated by the arrow is observed.

From a fundamental point of view, a study of plasmon waveguides provides insight into the nature of optical nearfields and electromagnetic light-matter interactions on the nanoscale. The strong dipolar coupling between metal 
nanoparticles can also serve as a model system for the study of other dipole-coupled energy transfer structures such as quantum dot chains ${ }^{20}$, magnetic nanoparticle arrays ${ }^{21}$, and coupled-resonator optical waveguides ${ }^{22}$.

In this paper, we present results of our efforts to model and understand the properties of ordered arrays of noble metal nanoparticles and assess their potential use as plasmon waveguides for electromagnetic energy transport at optical frequencies below the diffraction limit of light. The energy transport parameters such as group velocity and energy attenuation length are determined from finite-difference time-domain (FDTD) simulations of short arrays of spherical noble metal nanoparticles. The optical properties of silver nanoparticle plasmon waveguides fabricated using electron beam lithography are investigated, and a scheme for observing energy transport along the nanoparticle arrays is presented. Further information about our efforts to characterize plasmon waveguides ${ }^{23-26}$ and experimental evidence for energy transport over distances of about $500 \mathrm{~nm}^{27}$ can be found in the literature.

\section{THE SURFACE PLASMON RESONANCE}

\subsection{Position of the dipole surface plasmon resonance:}

It is well established that single noble metal nanoparticles interact strongly with visible light when resonantly excited at their surface plasmon frequency. The resonant behavior is due to the confinement of the conduction electrons inside the particle which sets up an effective restoring force due to surface polarization upon a light-induced displacement of the conduction electrons. For small particles $(D<15 \mathrm{~nm}$ for $\mathrm{Ag}$ and $D<25 \mathrm{~nm}$ for Au particles) retardation of the driving light field along the particle volume is negligible and all conduction electrons are excited inphase in a dipole-like oscillation. Quasistatic Mie-theory allows for the calculation of the far-field extinction cross section $\sigma_{\text {ext }}$ for a spherical metal nanoparticle of volume $V_{0}$ and dielectric function $\varepsilon(\omega)=\varepsilon_{1}(\omega)+i \varepsilon_{2}(\omega)$ embedded in a non-absorbing medium with dielectric constant $\varepsilon_{\mathrm{m}}$ :

$$
\sigma_{\mathrm{ext}}=9 \frac{\omega}{c} \varepsilon_{m}^{\frac{3}{2}} V_{0} \frac{\varepsilon_{2}(\omega)}{\left[\varepsilon_{1}(\omega)+2 \varepsilon_{m}\right]^{2}+\varepsilon_{2}(\omega)^{2}}
$$

For small damping $\left(\varepsilon_{2}(\omega), \frac{\partial \varepsilon_{2}}{\partial \omega}<<1,\right)$ the resonance occurs when the Fröhlich condition

$$
\varepsilon_{1}(\omega)=-2 \varepsilon_{m}
$$

is met. For small damping, the resonance position is only dependent on the real part $\varepsilon_{1}$ of the metal dielectric function and can be shifted throughout the visible and near-infrared range by embedding the particle in hosts with different dielectric constants $\varepsilon_{\mathrm{m}}$. For non-spherical particles in the dipole limit $(D<<\lambda)$, the resonance condition (2) is modified to account for the change in boundary conditions at the particle surface. For spheroids, this results in short-axes and longaxes dipole plasmon modes which are shifted with respect to the plasmon dipole resonance frequency of a spherical particle (Figure 2). Let us note that whereas for free electron metals the dipole resonance frequency $\omega_{0}$ depends only on the real part $\varepsilon_{1}(\omega)$ of the dielectric function and the surrounding medium $\varepsilon_{\mathrm{m}}$, and the resonance width only on $\varepsilon_{2}(\omega)$, for realistic metals both resonance position and width in general depend on $\varepsilon_{1}$ and $\varepsilon_{2}$. 


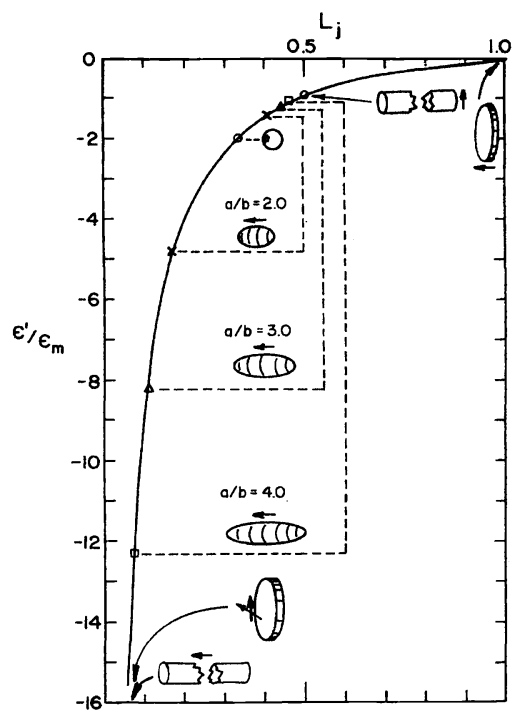

Figure 2. Dipole resonance position for spheroids with different aspect ratios 1:1, 2:1, 3:1 and 4:1 for both the long- and the short-axis mode of excitation. After ref ${ }^{11}$.

\subsection{Plasmon damping:}

The damping of a surface plasmon resonance of a metal nanoparticle is due to the dephasing of the coherent conduction electron motion with time. Figure 3 shows a schematic of the different processes involved in the damping of a surface plasmon excitation excited by a photon of energy $\mathrm{h} v$.

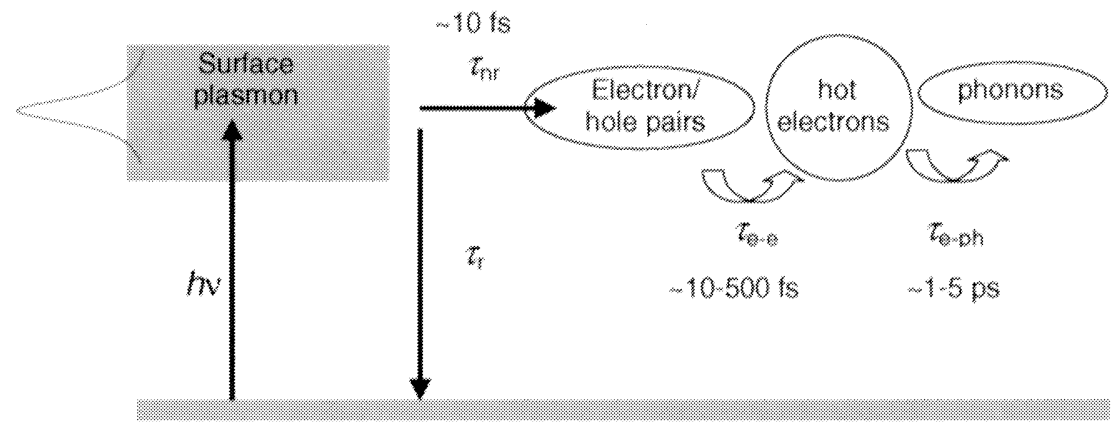

Figure 3. Energy relaxation of a surface plasmon. The relaxation takes place either via radiative or non-radiative processes. The nonradiative relaxation channel leads to the creation of electron-hole pairs and the subsequent relaxation into hot electrons and phonons.

The damping of the plasmon resonance is in general described via a total dephasing time $T_{2}$ and an energy relaxation time $T_{1}$. Both are related to the homogeneous linewidth $\Gamma$ of the surface plasmon resonance via

$$
\Gamma(\omega)=\frac{2}{T_{2}}=\frac{1}{T_{1}}+\frac{1}{T_{2}^{*}} .
$$

$T_{2}{ }^{*}$ is called the "pure dephasing time" which describes quasi-elastic electron scattering events that change the electron wavevector but not its energy. Its contribution to the total dephasing time $T_{2}$ is often put into the energy relaxation time 
$T_{1}$, yielding $T_{2}=2 T_{1}$. The damping of the plasmon resonance is thus determined by the energy relaxation time $T_{1}$, defined as

$$
\frac{1}{T_{1}}=\frac{1}{\tau_{n r}}+\frac{1}{\tau_{r}}
$$

The energy relaxation of a plasmon oscillation is composed of a non-radiative decay channel with timeconstant $\tau_{\text {nr }}$ and a radiative decay channel with timeconstant $\tau_{\mathrm{r}}$. For metal nanoparticles with a diameter $\mathrm{D}<<\lambda$ the energy relaxation is mostly due to non-radiative processes $\left(\tau_{\mathrm{r}} \gg \tau_{\mathrm{nr}}\right)$. The oscillating electrons loose their energy via inelastic scattering to single electron excitations (Landau damping), so called electron-holes states with a timeconstant $\tau_{\mathrm{nr}}$ on the order of $10 \mathrm{fs}$. These transitions can either be intraband or interband, and increase with increasing spectral overlap of the plasmon resonance with the interband absorption edge. The electron-hole states subsequently relax via electron-electron and electron-phonon scattering on the $100 \mathrm{fs}$ and $1 \mathrm{ps}$ timescale.

The plasmon damping of noble metal nanoparticles with diameters $5 \mathrm{~nm}<D<30 \mathrm{~nm}$ is mostly due to non-radiative processes and can thus be expressed via the materials parameters such as the optical conductivity or the dielectric function. Kreibig derived the following analytical approximation for the linewidth $\Pi(\omega)$ based on the optical conductivity as the only damping mechanism ${ }^{10}$ :

$$
\Gamma(\omega)=\frac{2 \varepsilon_{2}(\omega)}{\sqrt{\left(\frac{\partial \varepsilon_{1}}{\partial \omega}\right)^{2}+\left(\frac{\partial \varepsilon_{2}}{\partial \omega}\right)^{2}}}(1+\eta)
$$

where usually $\eta \ll 1$ holds. For free-electron metals described via a Drude model, $\Gamma$ reduces to the Drude relaxation frequency $\gamma$. During many studies, it has been revealed that plasmon decay times $T_{1}<10 \mathrm{fs}$ can be expected for Au and $\mathrm{Ag}$ nanoparticles throughout the visible regime. This will limit the maximum size of resonantly excited waveguides based on plasmon-polariton excitations in noble metal nanoparticles to the submicron regime.

\section{SIMULATIONS AND CHARACTERIZATION OF PLASMON WAVEGUIDES}

\subsection{Simulations of plasmon waveguides:}

Finite-difference time-domain (FDTD) simulations solve the full set of Maxwell's equations on a grid and are a powerful method to model light-matter interactions in complex systems ${ }^{28}$. Using FDTD simulations, we analyzed the near-field interactions of regularly spaced Au nanoparticles in air with an interparticle spacing $d<<\lambda$, and direct evidence for optical pulse propagation in locally excited plasmon waveguides was obtained.

The propagation of pulses through plasmon waveguides consisting of $50 \mathrm{~nm}$ Au spheres with a center-to-center spacing of $75 \mathrm{~nm}$ in air was examined by driving a local dipole source placed before the first particle with a pulse centered at the resonance energy $E_{0}=2.4 \mathrm{eV}$ of a single metal nanoparticle, corresponding to the $\mathbf{k}=\pi / 2 d$ waveguide mode with the highest group velocity ${ }^{26}$. The width of the pulses was chosen to be $95 \%$ of the bandwidth of the dispersion relation for each polarization in order to keep the pulse length of $30 \mathrm{fs}$ well within the total simulation time of $125 \mathrm{fs}$. The upper inset of Figure 4 shows a snapshot of the x-component of the electric field in the xy-plane for longitudinal polarization on a linear color scale. The periodicity of the field distribution along the chain confirms that the pulse is centered around the $\mathbf{k}=\pi / 2 d$ mode corresponding to a wavelength of 4 particle spacings. An analogous snapshot for the y-component of the electric field for transverse polarization is shown in the lower inset. The main part of Figure 4 shows the pulse position, defined as the location of maximum field amplitude, over time for both longitudinal (black squares) and transverse (black triangles) excitation. Linear fits of the datasets yield values for the 
group velocities of $v_{g L}^{*}=1.7 \times 10^{7} \mathrm{~m} / \mathrm{s}$ and $v_{g T}^{*}=5.7 \times 10^{6} \mathrm{~m} / \mathrm{s}$, in good agreement with the estimates $v_{g L}$ and $v_{g T}$ from a point-dipole model ${ }^{25}\left(v_{g L}=0.94 \times v_{g L}^{*}\right.$ and $\left.v_{g T}=1.15 \times v_{g T}^{*}\right)$.

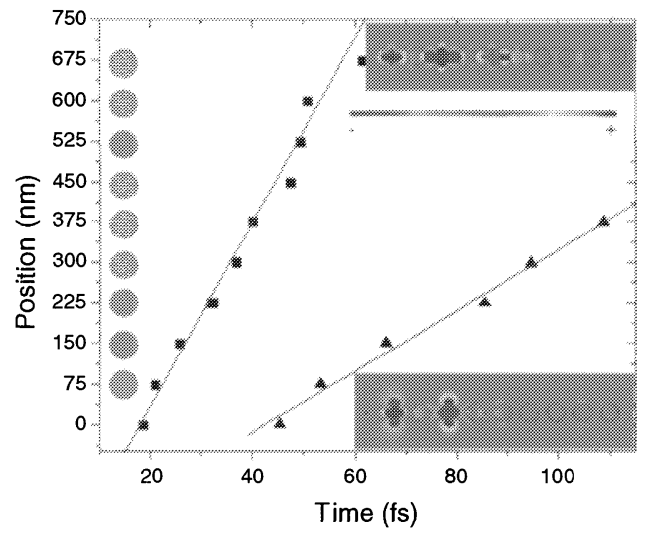

Figure 4: Pulsed local excitation of plasmon waveguides. The plot shows the pulse peak positions over time in a plasmon waveguide consisting of spherical particles for both longitudinal (black squares) and transverse (black triangles) polarization. The spheres along the ordinate indicate the position of the Au nanoparticles. Snapshots of the $\mathrm{x}(\mathrm{y})$ - component of the electric field in the xy-plane for longitudinal (transverse) polarization are shown in the upper (lower) inset.

The energy decay lengths can be obtained from the pulse propagation simulations by monitoring the pulse height expressed as the maximum field amplitude at the center of each nanoparticle as shown in Figure 5 for longitudinal polarization (black line). The energy decay lengths are $\alpha_{L}^{*}=6 \mathrm{~dB} / 280 \mathrm{~nm}$ and $\alpha_{T}^{*}=6 \mathrm{~dB} / 86 \mathrm{~nm}$ for longitudinal and transverse polarization, respectively. Both are between a factor 2-3 higher than the energy decay lengths calculated using the point-dipole model with a plasmon decay time $\tau=4 \mathrm{fs}{ }^{25}$. This discrepancy may be due to the finite size of our waveguide, resulting in a discrete spectrum of allowed modes. Nevertheless, these FDTD calculations quantitatively confirm the possibility of using plasmon waveguides for information transport with $v_{g}>0.01 \mathrm{c}$. Figure 5 also shows results for a plasmon waveguide with the first two particles after the source was removed (red dataset), resulting in a decreased pulse height at the last particle by about a factor 10. This directly confirms that the guiding is due to particle interactions.

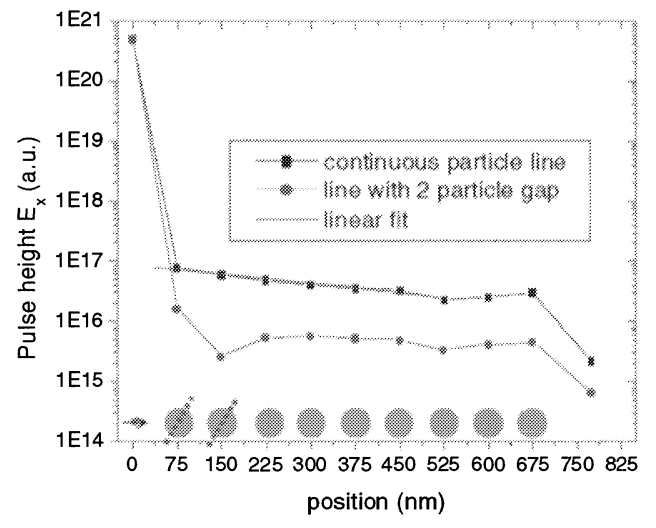

Figure 5: Energy decay during pulse propagation in plasmon waveguides. The plot shows the maximum field amplitudes of a longitudinally polarized pulse monitored at the source position and at the center of each nanoparticle (black line) traveling through a plasmon waveguide. Also shown is data for a plasmon waveguide with the first two particles removed (red line), resulting in a decreased pulse height inside the last particle. The initial drop after the source is partly due to the point-dipole-like nature of the source. 
The previous analysis dealt exclusively with spherical particles. The energy guiding properties of plasmon waveguides can be optimized by a change in particle geometry to non-spherical particles as suggested by calculations based on extensions of the Mie-theory to ellipsoidal particles ${ }^{25}$. Rod-shaped nanoparticles with their long axis oriented perpendicular to the waveguide chain axis show an increased interparticle coupling compared to spherical particles that have the same particle volume and center-to-center spacing, as shown in reference ${ }^{25}$. Additionally, gold nanorods with an aspect ratio of 3:1 were shown to exhibit an increase in plasmon decay time $\tau$ by about a factor 2-3 due to a resonance shift away from the interband transition edge ${ }^{29}$. The waveguide properties of metal nanoparticle arrays can thus be optimized by tuning the particle geometry, and group velocities for energy transport of about $0.1 c$ should be achievable.

\subsection{Local excitation of plasmon waveguides:}

The transport properties of plasmon waveguides can also be numerically examined in FDTD simulations as described above by using a local dipole excitation source placed in close proximity to the nanoparticle arrays instead of plane-wave illumination. This way, all modes of the dispersion relation are accessible. Figure 6 shows an example of a waveguide consisting of $950 \mathrm{~nm}$ Au particles with a center-to-center spacing $d=75 \mathrm{~nm}$ in air. A point-dipole polarized in the $\mathrm{x}$-direction (longitudinal mode) and driven at the resonance frequency $\omega_{0}$ of a single Au nanoparticle is placed 75 $\mathrm{nm}$ to the left of the nanoparticle array, providing thus a continuous local excitation source. Figure 6 shows the $\mathrm{x}$ component of the electric field around the plasmon waveguide structure $60 \mathrm{fs}$ after starting the continuous excitation. A mode profile with $\mathbf{k}=\pi / 2 d$ is clearly visible along the nanoparticle structure, in agreement with the dispersion relation calculated using the analytical point-dipole model discussed above.

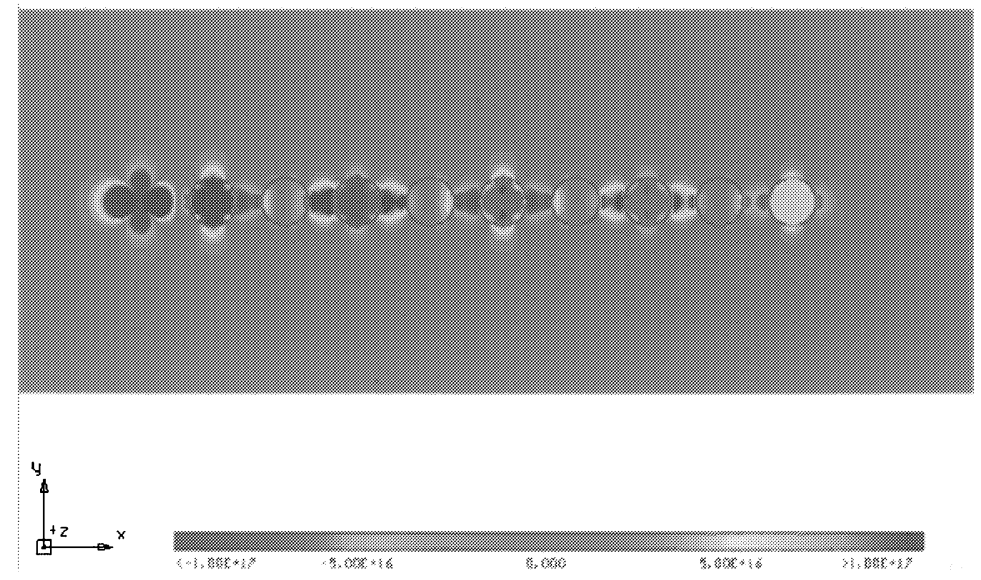

Figure 6. Finite-difference time-domain simulation of a plasmon waveguide consisting of $950 \mathrm{~nm}$ Au particles spaced $75 \mathrm{~nm}$ apart. The particles are locally excited by a point dipole with longitudinal polarization placed $75 \mathrm{~nm}$ to the left of the nanoparticle array. The dipole frequency is set equal to the plasmon resonance frequency of a single nanoparticle, thus exciting the plasmon waveguide in the $\mathbf{k}=\pi / 2 d$ mode in agreement with the predictions from the point-dipole dispersion relation.

The plasmon waveguides used in this analysis of energy transport consisted of rod-shaped Ag nanoparticles with dimensions of $90 \mathrm{~nm} \times 30 \mathrm{~nm} \times 30 \mathrm{~nm}$ and a surface-to-surface spacing of $50 \mathrm{~nm}$ between adjacent particles. The long axes of the individual nanoparticles were oriented perpendicular to the waveguide chain axis in order to allow for an increased near-field coupling between the particles as described in ${ }^{25}$. The structures were fabricated using electron beam lithography with lift-off on ITO coated quartz slides, which allowed for a good control over particle size and spacing. The inset of Figure 7 shows a scanning electron micrograph of a plasmon waveguide of this geometry. In order to determine the plasmon resonances of the fabricated structures with a high signal-to-noise ratio using far-field spectroscopy, a large number of plasmon waveguides were arranged in a $100 \mu \mathrm{m}$ x $100 \mu \mathrm{m}$ grid with a grating constant of $1 \mu \mathrm{m}$ as depicted in Figure 8b. Cross talk between different waveguides is negligible for this grating constant ${ }^{24}$. This way, far-field extinction spectra on these arrays probe the near-field coupling between the nanoparticles comprising each waveguide only and reflect thus the properties of individual plasmon waveguides. 


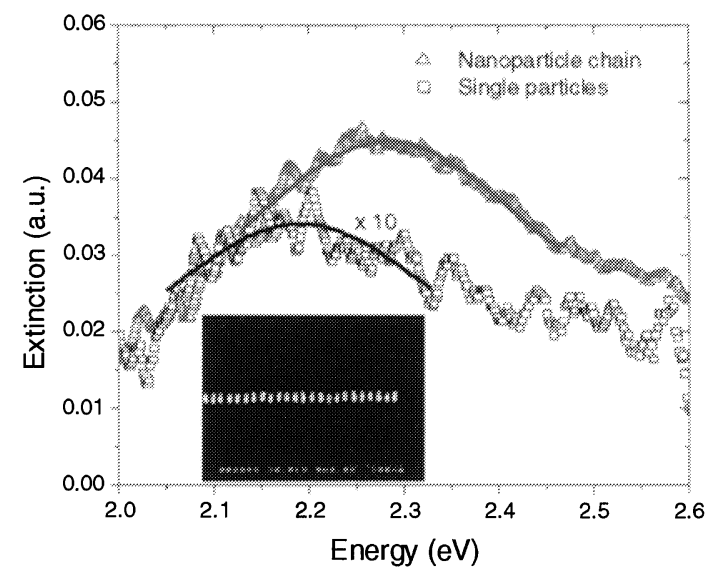

Figure 7: Far-field extinction spectrum of $\mathrm{Ag}$ nanoparticle chains and non-interacting particles. The far-field extinction spectrum of a plasmon waveguide consisting of Ag nanorods with a 3:1 aspect ratio and a surface-to-surface spacing of $50 \mathrm{~nm}$ between adjacent particles shows a plasmon resonance peak shift to higher energies (red triangles and Lorentz fit) compared to the extinction spectrum of isolated, non-interacting particles (black squares and Lorentz fit). The exciting light was polarized along the long axis of the nanorods, perpendicular to the particle chain axis. The inset shows an SEM micrograph of the plasmon waveguide under study.

Figure 7 shows the far-field extinction spectrum of the fabricated plasmon waveguides taken under normal incidence white light illumination with a spot size of $100 \mu \mathrm{m}$ and a polarization along the long axis of the nanoparticles and thus perpendicular to the waveguide chain axis (red data points). Also shown is the extinction spectrum of a grid of single Ag nanoparticles of the same geometry with an interparticle spacing of $1 \mu \mathrm{m}$, for which the interparticle coupling is negligible (black data points). The total area density of nanoparticles is lower in this case, leading to an overall smaller signal. The single particle extinction spectrum peaks at $2.18 \mathrm{eV}$ for a polarization along the long particle axis, corresponding to a resonance wavelength of $570 \mathrm{~nm}$. The extinction spectrum of the plasmon waveguide shows a resonance shift of about $100 \mathrm{meV}$ to higher energies due to near-field coupling between the particles, in agreement with the numerical simulations ${ }^{25}$. According to these simulations, a resonance shift of $100 \mathrm{meV}$ should translate into a maximum energy attenuation length on the order of $6 \mathrm{~dB} / 200 \mathrm{~nm}$ for an excitation at the single particle resonance at $2.18 \mathrm{eV}$, at which the energy transfer in a plasmon waveguide is most efficient.

In order to directly observe signs of energy transport in the fabricated plasmon waveguides, a local excitation of a non-zero wave vector $\mathbf{k}$ is necessary as opposed to an in-phase excitation of the particle arrays as a whole at $\mathbf{k}=0$ using far-field illumination. To accomplish this, the tip of an illumination mode near-field optical microscope (Nanonics NSOM-100) was used as a local excitation source for nanoparticles in plasmon waveguides. Light from a dye laser at a wavelength of $570 \mathrm{~nm}$, corresponding to the single particle resonance, was used in order to excite the mode of least damping. The light was coupled into a multimode optical fiber attached to the Al-coated NSOM tip used for excitation. Figure 8a shows a schematic of our principle of excitation and energy transport detection.

a)

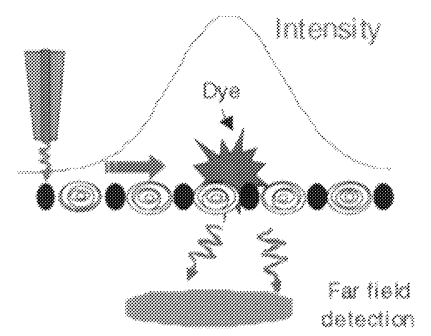

b)

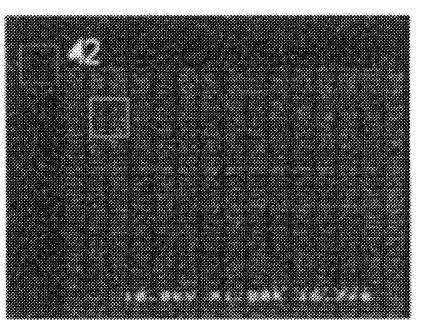

Figure 8: Near-field optical microscopy excitation and energy transport detection of plasmon waveguides. a) Sketch of the experiment. Light emanating from the tip of an illumination-mode near-field optical microscope (NSOM) locally excites a plasmon 
waveguide. The waveguide guides the electromagnetic energy to a fluorescent nanosphere, and the fluorescence intensity for varying tip positions is collected in the far-field. b) SEM micrograph of a $100 \times 100 \mu \mathrm{m}$ grid consisting of $\mathrm{Ag}$ plasmon waveguides.

Power transport away from the directly excited nanoparticles of the plasmon waveguide is probed via the placement of polystyrene nanospheres filled (Molecular Probes Fluospheres F-8801, diameter 110 \pm 8 nm) with fluorescent molecules in close proximity to the waveguide structure. For this, the electron beam fabricated plasmon waveguide sample was coated with a thin poly-lysine layer, and the nanospheres were subsequently randomly deposited from an aqueous solution. The fluorescent dyes used show a strong absorption peaking at 580-590 nm near the plasmon resonance wavelength of a single fabricated $\mathrm{Ag}$ particle and emit radiation peaking at $610 \mathrm{~nm}$, which is detected in the far-field using a band pass filter and an avalanche photodiode. This scheme enables the observation of energy transport in the following way: first, energy is transferred from the illuminating tip to the plasmon waveguide. The excitation subsequently propagates along the nanoparticle structure and excites a fluorescent nanosphere placed on top of a waveguide at a sufficient distance from the excitation source. Energy transport would result in dye emission even when the tip is located away from the dye, and thus would manifest itself in an increased spatial width of the fluorescence spot of a nanosphere attached to a plasmon waveguide compared to a single free nanosphere.

The left panel of Figure 9 shows the spatial intensity variation in the waveguide direction of fluorescent nanospheres located at a distance (A, B) and on top of plasmon waveguides (WG1, WG2). Gaussian fits to the data for both the control spheres (black and red lines and data points) and the two spheres attached to plasmon waveguides (green and blue lines and data points) are included. The control nanospheres A and B were scanned before and after scanning the waveguide structures, and in both cases show similar fluorescent full widths at half maximum (FWHM) of $174 \pm 17$ and $193 \pm 23 \mathrm{~nm}$. Analyses of additional control spheres yielded similar widths of $160 \pm 12,205 \pm 13$, and $188 \pm 17 \mathrm{~nm}$, respectively. This confirms that the tip profile did not change appreciably during the scans. By contrast, the fluorescent spots WG1 and WG2 of the nanospheres attached to the plasmon waveguides show a FWHM of $329 \pm 14$ and $343 \pm 27$ $\mathrm{nm}$, respectively. The resulting average fluorescence spot FWHM of control spheres is $185 \pm 38 \mathrm{~nm}$, while the average width of nanospheres on waveguides is $336 \pm 30 \mathrm{~nm}$. The broadening of the fluorescence spots due to the presence of the plasmon waveguide is thus $151 \pm 48 \mathrm{~nm}$.
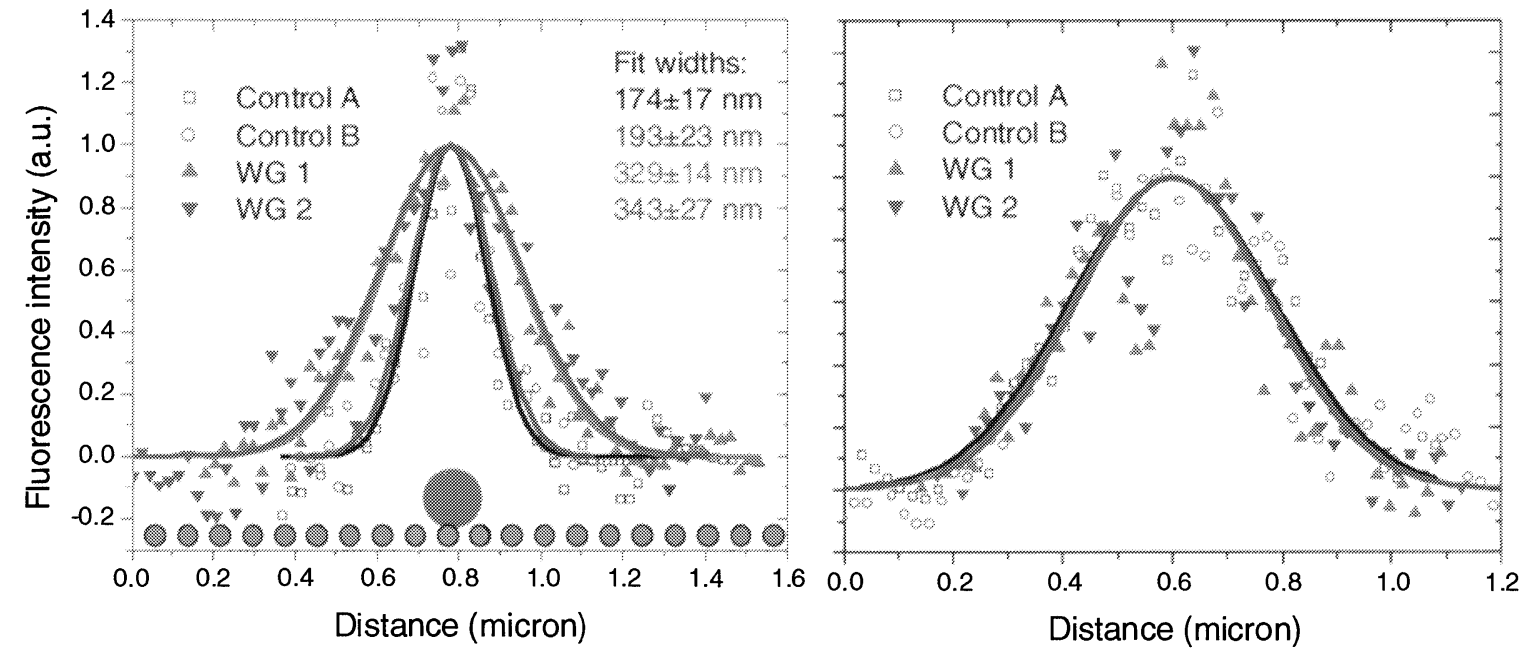

Figure 9: Evidence for energy transport in plasmon waveguides via the width of the fluorescence intensity of fluorescent nanospheres. Nanospheres WG1 and WG2 located on top of plasmon waveguides show an increased spatial width in the waveguide direction only compared to single nanospheres A and B located at a distance (left graph). Perpendicular to the waveguide direction, no broadening due to the presence of the plasmon waveguide is observed (right graph).

If the observed broadening of the fluorescent width of waveguide nanospheres is due to transport of electromagnetic energy along the waveguide, no difference in FWHM between control and waveguide nanospheres is expected in the direction perpendicular to the waveguide axis. Indeed, the fitted FWHMs in this direction are found to be equal within 
the fit error, giving $364 \pm 36 \mathrm{~nm}$ for control A and B and $352 \pm 40 \mathrm{~nm}$ for the waveguide nanospheres WG1 and WG2 as shown in Figure 9 (right panel). Note that these values are rather large due to the shape of the tip aperture.

The increase in the width of the nanosphere fluorescence of about $150 \mathrm{~nm}$ for spheres attached to a plasmon waveguide structure can be attributed to local excitation of the plasmon waveguide followed by energy transport along the waveguide toward the fluorescent dye particle. This type of excitation is known to dominate over excitation by direct scattering of the exciting radiation from individual nanoparticles ${ }^{25}$. From Figure 9 it is clear that for a freestanding nanosphere the fluorescence signal decreases below the dark noise level if the tip is located approximately $200 \mathrm{~nm}$ away from the sphere center, while a fluorescent nanosphere attached to a plasmon waveguide can be excited from distances up to $500 \mathrm{~nm}$ via the plasmon waveguide. Our results thus provide direct evidence for energy transport over this distance. To obtain quantitative information on the energy decay length we have performed exponential fits on the tails of the fluorescence intensity of the waveguide nanospheres at distances $>200 \mathrm{~nm}$ away from the nanosphere center. At this distance excitation via the plasmon waveguide dominates over direct dye excitation. These fits yield a decay length of $6 \mathrm{~dB} / 195 \pm 28 \mathrm{~nm}$, which is in excellent agreement with the estimate of $6 \mathrm{~dB} / 200 \mathrm{~nm}$ obtained from our far-field measurements and theoretical predictions.

\section{CONCLUSIONS}

Using both numerical finite-difference time-domain simulations and near-field optical experiments, we have firmly established the possibility of using arrays of closely spaced metal nanoparticles as plasmon waveguides for the transport of electromagnetic energy below the diffraction limit of light. Such waveguides could be used as energy concentrators attached to conventional dielectric waveguides to channel energy to nanoscale or molecular detectors and allow for the fabrication of highly integrated optical circuits.

\section{ACKNOWLEDGEMENTS}

This work was supported by the Air Force Office of Scientific Research and the Center for Science and Engineering of Materials at Caltech (NSF).

\section{REFERENCES}

B. E. A. Saleh and M. C. Teich, Fundamentals of Photonics. (Wiley, New York, 1991).

Attila Mekis, J. C. Chen, I. Kurland et al., "High transmission through sharp bends in photonic crystal waveguides," Physical Review Letters 77 (18), 3787-3790 (1996); Jürgen Moosburger, Martin Kamp, Alfred Forchel et al., "Enhanced transmission through photonic-crystal-based bent waveguides by bend engineering," Applied Physics Letters 79 (22), 3579-3581 (2001). O. Painter, R. K. Lee, Axel Scherer et al., "Two-dimensional photonic band-gap defect mode laser," Science 284 (5421), 1819-1821 (1999). Heinz Raether, Surface Plasmons on smooth and rough surfaces and on gratings. (Springer, Berlin, 1988). J. Tominaga, C. Mihalcea, D. Büchel et al., "Local plasmon photonic transistor," Applied Physics Letters 78 (17), 2417-2419 (2001). Jörg P. Kottmann and Olivier J. F. Martin, "Plasmon resonant coupling in metallic nanowires," Optics Express 8 (12), 655-663 (2001); Jörg P. Kottmann, Olivier J. F. Martin, David R. Smith et al., "Plasmon resonances of silver nanowires with a nonregular cross section," Physical Review B 64, 235402 (2001).

7 B. Lamprecht, J. R. Krenn, G. Schider et al., "Surface plasmon propagation in microscale metal stripes," Applied Physics Letters 79 (1), 51-53 (2001). J. C. Weeber, A. Dereux, C. Girard et al., "Plasmon polaritons of metallic nanowires for controlling submicron propagation of light," Physical Review B 69 (12), 9061-9068 (1999); Robert M. Dickson and L. Andrew Lyon, "Unidirectional plasmon propagation in metallic nanowires," Journal of Physical Chemistry B 104, 6095-6098 (2000). Junichi Takahara, Suguru Yamagishi, Hiroaki Taki et al., "Guiding of a one-dimensional optical beam with nanometer diameter," Optics Letters 22 (7), 475-477 (1997). U. Kreibig and M. Vollmer, Optical properties of metal clusters. (Springer, Berlin, 1995). 

901-903 (1995).

\footnotetext{
*e-mail: stmaier@caltech.edu
}

Craig F. Bohren and Donald R. Huffman, Absorption and scattering of light by small particles. (Wiley, New York, 1983).

T. A. Klar, M. Perner, S. Grosse et al., "Surface-plasmon resonances in single metallic particles," Physical Review Letters 80 (19), 4249-4252 (1998).

M. Quinten, A. Leitner, J. R. Krenn et al., "Electromagnetic energy transport via linear chains of silver nanoparticles," Optics Letters 23 (17), 1331-1333 (1998).

Mark L. Brongersma, John W. Hartman, and Harry A. Atwater, "Electromagnetic energy transfer and switching in nanoparticle chain arrays below the diffraction limit," Physical Review B 62, R16356 (2000).

H. G. Craighead and G. A. Niklasson, "Characterization and optical properties of arrays of small gold particles," Applied Physics Letters 44 (12), 1134-1136 (1984).

J. P. Hoogenboom, D. L. J. Vossen, C. Faivre-Moskalenko et al., "Patterning surfaces with colloidal particles using optical tweezers," Applied Physics Letters 80 (25), 4828-4830 (2002).

R. Andrew McMillan, Chad D. Paavola, Jeanie Howard et al., "Ordered nanoparticle arrays formed on engineered chaperonin protein templates," Nature Materials 1, 247-252 (2002).

T. Müller, K.-H. Heinig, and B. Schmidt, "Template-directed self-assembly of buried nanowires and the pearling instability," Materials Science and Engineering C 19, 209-213 (2002).

J.J. Penninkhof, A. Polman, L.A. Sweatlock et al., "MeV ion beam induced anisotropic plasmon resonance of silver nanocrystals in glass," Applied Physics Letters 83 (4137) (2003).

D. S. Citrin, "Coherent transport of excitons in quantum-dot chains: role of retardation," Optics Letters 20 (8),

Pankaj Poddar, Tamar Telem-Sharif, Tcipi Fried et al., "Dipolar interactions in two- and three-dimensional magnetic nanoparticle arrays," Physical Review B 66, 060403(R) (2002).

Amnon Yariv, Yong Xu, Reginald K. Lee et al., "Coupled-resonator optical waveguide: a proposal and analysis," Optics Letters 24 (11), 711-713 (1999).

Stefan A. Maier, Mark L. Brongersma, and Harry A. Atwater, "Electromagnetic energy transport along arrays of closely spaced metal rods as an analogue to plasmonic devices," Applied Physics Letters 78 (1), 16-18 (2001); Stefan A. Maier, Mark L. Brongersma, and Harry A. Atwater, "Electromagnetic energy transport along Yagi arrays," Materials Science and Engineering C 19, 291-294 (2002); Stefan A. Maier, Mark L. Brongersma, and Harry A. Atwater, in MRS Proceedings (2001), Vol. 637, p. E2.9; Stefan A. Maier, Mark L. Brongersma, Pieter G. Kik et al., "Plasmonics - a route to nanoscale optical devices," Advanced Materials 13 (19), 15011505 (2001); Stefan A. Maier, Pieter G. Kik, and Harry A. Atwater, "Observation of coupled plasmon-polariton modes in Au nanoparticle chain waveguides of different lengths: Estimation of waveguide loss," Applied Physics Letters 81, 1714-1716 (2002); Stefan A. Maier, Pieter G. Kik, Harry A. Atwater et al., in Proceedings of SPIE (2002), Vol. 4810, pp. 71-82; Stefan A. Maier, Pieter G. Kik, Mark L. Brongersma et al., in Proceedings of SPIE (2001), Vol. 4456, pp. 22-30.

Stefan A. Maier, Mark L. Brongersma, Pieter G. Kik et al., "Observation of near-field coupling in metal nanoparticle chains using far-field polarization spectroscopy," Physical Review B 65, 193408 (2002).

Stefan A. Maier, Pieter G. Kik, and Harry A. Atwater, "Optical pulse propagation in metal nanoparticle chain waveguides," Physical Review B 67, 205402 (2003).

Stefan A. Maier, Pieter G. Kik, Mark L. Brongersma et al., in MRS Proceedings (2002), Vol. 705, p. Y3.6. Stefan A. Maier, Pieter G. Kik, Harry A. Atwater et al., "Local detection of electromagnetic energy transport below the diffraction limit in metal nanoparticle plasmon waveguides," Nature Materials 2, 229 (2003). Kurt L. Shlager and John B. Schneider, "A selective survey of the finite-difference time-domain literature," IEEE Antennas \& Propagation Magazine 37 (4), 39-56 (1995).

C. Sönnichsen, T. Franzl, T. Wilk et al., "Drastic reduction of plasmon damping in gold nanorods," Physical Review Letters 88 (7), 077402 (2002). 